Case Report

\title{
A Rare Case of Systemic Sarcoidosis in a Middle-Aged Female with a Solitary Thyroid Nodule
}

\author{
Ayrton Bangolo $\mathbb{D}^{1},{ }^{1}$ John Bukasa Kakamba ${ }^{(D,},{ }^{2,3}$ Ali Atoot, ${ }^{4}$ Mohammad Jurri, ${ }^{5}$ \\ Ashraf Mahmoud, ${ }^{5}$ Arthur Oliver Lo, ${ }^{6}$ Kunchang Song, ${ }^{7}$ Syed Sirajuddin, ${ }^{4}$ and Adam Atoot ${ }^{1}$ \\ ${ }^{1}$ Department of Internal Medicine, Hackensack Meridian Health Palisades Medical Center, North Bergen, NJ, USA \\ ${ }^{2}$ Endocrine and Metabolic Disorders Department, University Clinics of Kinshasa, Kinshasa, Democratic Republic of the Congo \\ ${ }^{3}$ Department of Endocrinology, University of Liege, Liege, Belgium \\ ${ }^{4}$ Department of Anesthesia, Hackensack University Medical Center, Hackensack, NJ, USA \\ ${ }^{5}$ Department of Family Medicine, Hackensack Meridian Health Palisades Medical Center, North Bergen, NJ, USA \\ ${ }^{6}$ University of the East Ramon Magsaysay, Quezon City, Philippines \\ ${ }^{7}$ Department of Pathology, Hackensack Meridian Health Palisades Medical Center, North Bergen, NJ, USA
}

Correspondence should be addressed to Ayrton Bangolo; ayrtonbangolo@yahoo.com

Received 2 September 2021; Revised 22 November 2021; Accepted 9 December 2021; Published 21 December 2021

Academic Editor: Mihail A. Boyanov

Copyright (c) 2021 Ayrton Bangolo et al. This is an open access article distributed under the Creative Commons Attribution License, which permits unrestricted use, distribution, and reproduction in any medium, provided the original work is properly cited.

\begin{abstract}
Sarcoidosis is a multisystem inflammatory disease characterized by the presence of noncaseating granulomas. Sarcoidosis can affect any organ of the body, the lung being the most affected. Sarcoidosis rarely affects the thyroid gland, and only a few cases of thyroid-related sarcoidosis have been reported in the literature. Herein, we report a case of systemic sarcoidosis with multiple organ involvement in a patient with a solitary left thyroid nodule and benign Fine Needle Aspiration (FNA) findings. The patient was eventually diagnosed with thyroid sarcoidosis using a core needle biopsy of the thyroid nodule. With this case report, we highlight the limitations of the FNA. This case report has the objective to encourage clinicians to include thyroid sarcoidosis in the differential diagnosis of thyroid nodules in patients with systemic sarcoidosis even with unremarkable FNA findings.
\end{abstract}

\section{Introduction}

Sarcoidosis is a multisystem granulomatous disorder of unknown etiology that affects individuals worldwide and is characterized pathologically by the presence of noncaseating granulomas in involved organs [1]. Up to 30 percent of patients present with extrapulmonary sarcoidosis [2]. Approximately 8 percent of patients with sarcoidosis present with disease at extrapulmonary sites without lung involvement. In these patients, the skin is the most common site and accounted for nearly half of the patients [3]. Thyroid involvement results in diffuse goiter or solitary thyroid nodule [4]. Most patients with thyroid involvement remain euthyroid and only a few cases have been associated with hypothyroidism [5]. We report the case of a middle-aged female who presented for evaluation of hypercalcemia and was found to have systemic sarcoidosis and a solitary left thyroid nodule. The thyroid nodule was found to be unremarkable on Fine Needle Aspiration (FNA), but sarcoid infiltration of the gland was revealed on Core Needle Biopsy. With this case report, we aim to encourage physicians to include sarcoidosis in the differential diagnosis of thyroid nodules even with normal FNA and recognize the limitations of FNA.

\section{Case Presentation}

This is a 58-year-old female with a past medical history of Diabetes Mellitus and asthma who presented for evaluation of hypercalcemia. The patient was referred from the Primary Care Physician's office after routine blood work revealed elevated calcium levels. Of note, the patient visited her 


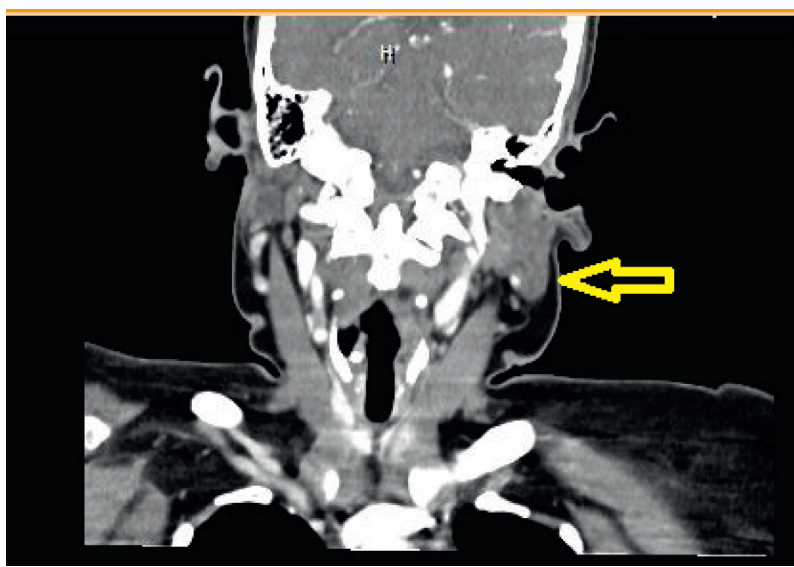

FIGURE 1: Neck soft tissue computed tomography revealing the left thyroid nodule.

TABLE 1: Laboratory values on admission.

Calcium

Aspartate aminotransferase

Alanine aminotransferase

Parathyroid hormone

Parathyroid hormone-related peptide

$25(\mathrm{OH})$ vitamin $\mathrm{D}$

$1,25(\mathrm{OH})_{2}$ vitamin D

Phosphorus

Thyroid-stimulating hormone Anti-thyroid peroxidase antibody

Anti-thyroglobulin antibody

Alkaline phosphatase (ALP)

Bone specific ALP

Gamma-glutamyl transferase

Angiotensin-1-converting enzyme
Laboratory values

14 milligram/deciliter (mg/dl) (8.6-10.3)

44 units/liter (U/L) (13-39)

$47 \mathrm{U} / \mathrm{L}(7-52)$

3 picogram/milliliter (pg/ml) (14-64)

$20 \mathrm{pg} / \mathrm{ml}(14-27)$

22.3 nanogram/milliliter (20-80)

$66 \mathrm{pg} / \mathrm{ml}(18-72)$

$3.6 \mathrm{mg} / \mathrm{dl}(3.0-4.3)$

2.87 milli-international units per milliliter $(0.450-4.50)$

9 international units per milliliter $(\mathrm{IU} / \mathrm{mL})(0-34)$

$<1.00 \mathrm{IU} / \mathrm{mL}(0.0-0.9)$

$323 \mathrm{U} / \mathrm{L}(34-104)$

$29.6 \mathrm{microgram} /$ milliliter (5.6-29)

$282 \mathrm{U} / \mathrm{L}(9-64)$

$115 \mathrm{U} / \mathrm{L}(9-67)$

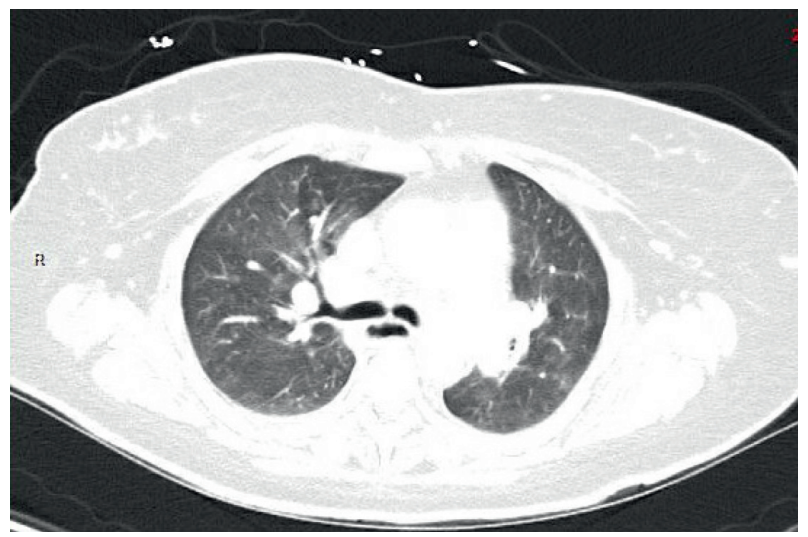

FIGURE 2: Chest computed tomography revealing mediastinal and hilar lymphadenopathy.

primary care physician for evaluation of cough and dyspnea one month prior; encounter during which a chest roentgenogram revealed left lobe enlargement with right tracheal deviation. She underwent a subsequent computed tomography $(\mathrm{CT})$ of neck soft tissue which revealed 3.5 centimeters

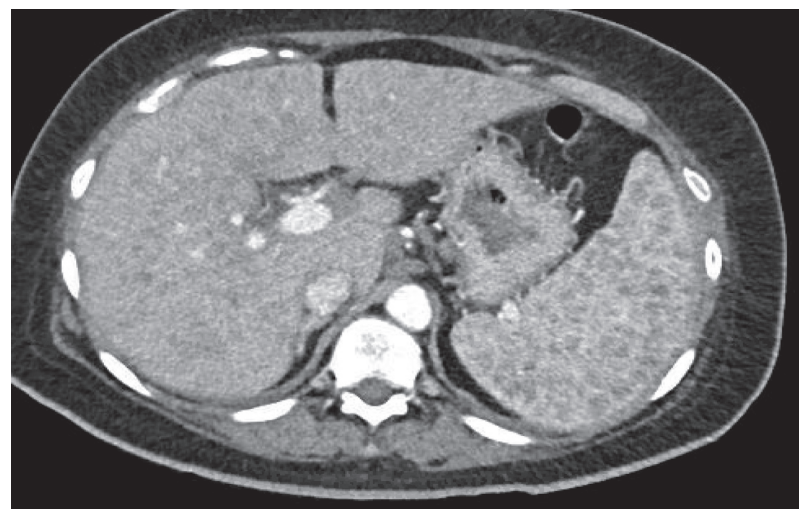

FIGURE 3: Computed tomography of the abdomen revealing multiple nodules of the liver and spleen.

nodule of the left thyroid gland (Figure 1). A fine needle aspiration of the nodule revealed a Bethesda category II, benign. However, the specimen was marginally adequate. She was also diagnosed with hypothyroidism and levothyroxine was initiated. She reported a globus sensation for the past 2 months prior to our encounter. She also reported 


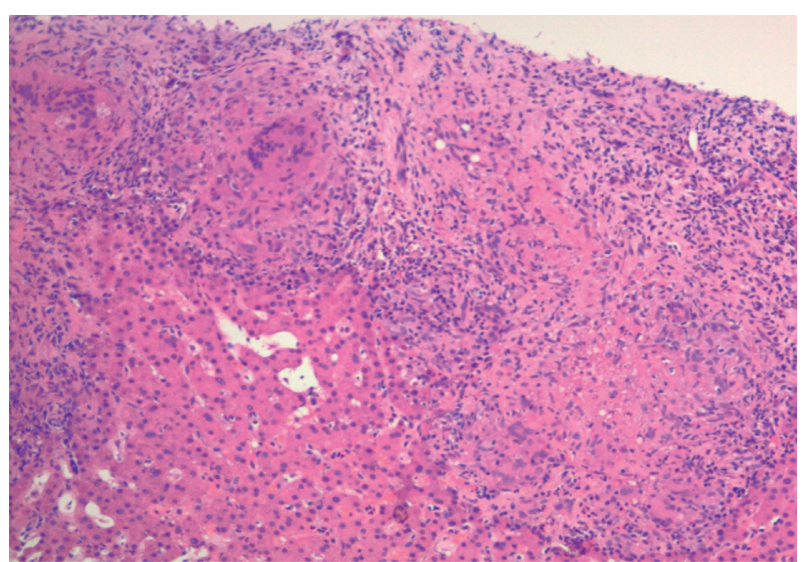

FIgURE 4: Thyroid nodule core biopsy pathology report revealing non-necrotizing granulomatous infiltration consistent with sarcoidosis. Trichrome stain showed no significant fibrosis, and iron stain was negative for iron deposition.

abdominal pain associated with constipation for the past week prior to our encounter. She denied any dysphagia or change in voice.

Upon arrival in the Emergency Department, she was hemodynamically stable. A left thyroid nodule with cervical lymphadenopathy could be felt on physical examination. Her laboratory results revealed an elevated serum calcium, higher normal $1,25(\mathrm{OH})_{2}$ vitamin $\mathrm{D}$ and lower normal $25(\mathrm{OH})$ vitamin $\mathrm{D}$, and an elevated Angiotensin Converting Enzyme level, as seen in Table 1. A CT of the chest and abdomen revealed mediastinal and hilar lymphadenopathy (Figure 2) and multiple nodules of the liver and spleen (Figure 3). A subsequent biopsy of the liver revealed granulomatous hepatitis consistent with sarcoidosis.

The patient was started on normal saline continuous infusion. Nephrology and oncology were consulted. The patient's calcium level had normalized on day 2 of hospitalization. She eventually underwent a core needle biopsy of the left thyroid nodule which revealed granulomatous infiltration of the gland consistent with sarcoidosis (Figure 4).

She was referred to Ear, Nose, and Throat for a thyroidectomy given the invasive nature of the nodule.

\section{Discussion}

Sarcoidosis is an inflammatory disease characterized by the presence of noncaseating granulomas. Although sarcoidosis can affect virtually every organ of the body, the lung is most affected. Other organs commonly affected are the liver, skin, and eye [6]. Sarcoidosis rarely affects the thyroid gland. Sarcoidosis can cause diffuse goiter or rarely solitary or multiple thyroid nodules [4] that resemble a malignancy, especially when associated with cervical adenopathy [7]. Although most patients are euthyroid, both hypothyroidism and hyperthyroidism have been reported [8]. Our patient's sarcoidosis has multiple organs involved including the thyroid gland, lymph nodes, liver, spleen, lungs, and mediastinum. She has a solitary thyroid nodule with lymphadenopathy; those findings can mimic a thyroid cancer with metastasis.

Respiratory complaints including cough and dyspnea are the most common presenting symptoms. Fatigue is the most common constitutional symptom [6]. The most common abnormality of liver function is an elevation of the alkaline phosphatase level, consistent with an obstructive pattern. In addition, elevated transaminase levels can occur [6]. Our patient was initially complaining of cough and dyspnea, but a chest roentgenogram failed to show characteristic features of sarcoidosis which delayed the diagnosis. She did not have any constitutional symptoms. The liver profile revealed a cholestatic pattern consistent with sarcoidosis. Hypercalcemia occurs in about $10 \%$ of sarcoidosis patients. The mechanism of abnormal calcium metabolism is increased production of 1, 25-dihydroxyvitamin $\mathrm{D}$ by the granuloma itself [6]. As seen in our patient, hypercalcemia was associated with higher normal $1,25(\mathrm{OH})_{2}$ vitamin $\mathrm{D}$ and a lower normal $25(\mathrm{OH})$ vitamin D. Several conditions can cause mild elevation of Angiotensin Converting Enzyme (ACE) but only a few conditions, including sarcoidosis, will cause an ACE elevation more than $50 \%$ of the upper limit of normal [6]. Elevated levels of ACE are reported in $60 \%$ of patients with acute disease and only $20 \%$ of patients with chronic disease [6]. Our patient had an ACE level elevation of more than $50 \%$ of the upper limit of normal, consistent with sarcoidosis.

Thyroid sarcoidosis is diagnosed with fine needle aspiration (FNA) biopsy or thyroidectomy for treatment of goiter demonstrating noncaseating epithelioid granulomas [9]. The false-negative rate of a benign interpretation is 0 to 3 percent [10]. In a retrospective study of nodules with two or more prior nondiagnostic FNAs, core needle biopsy was diagnostic in 86 compared with 29 percent for repeat FNA [11]. Core needle biopsy has low nondiagnostic result rates and high specificity for the diagnosis of malignancy. It is a safe diagnostic technique with a higher diagnostic yield, especially when molecular testing is not available or fine needle aspiration does not yield enough cells for molecular testing [12]. Our patient underwent an initial FNA with a marginally adequate cellularity; thus, a definitive diagnosis could not be obtained. A repeat FNA was warranted but given the higher specificity and sensitivity of the core needle biopsy compared to a repeat FNA; our patient underwent a core needle biopsy. The procedure revealed the final diagnosis.

Patients with sarcoidosis of the thyroid do not usually need treatment unless they have a goiter of sufficient size to cause obstructive symptoms or thyroid dysfunction. Thyroidectomy is generally performed in patients with obstructive symptoms or other absolute indications for surgery, such as thyrotoxicosis or thyroid cancer. Hypothyroidism, if present, should be treated with thyroid hormone therapy [9]. Our patient has dyspnea and tracheal deviation on imaging caused by a large thyroid nodule; thus, she was referred for surgery. The large nodule also caused hypothyroidism for which she is on levothyroxine. 


\section{Conclusion}

Sarcoidosis is a multisystem granulomatous disease that can affect any organ. Some organs are more affected than others. The thyroid gland is one of the least affected organs, and only a few cases of thyroid-related sarcoidosis have been reported in the literature. Fine needle aspiration (FNA) is an important diagnostic tool in the evaluation of thyroid nodules. However, FNA can have limitations in the diagnosis of certain conditions requiring more cells for molecular testing such as sarcoidosis. Core needle biopsy has low nondiagnostic results and should be used in case the FNA sample is marginally adequate. With this case report, we hope to contribute to the growing literature of thyroid-related sarcoidosis and encourage physicians to include such a condition in the differential of thyroid nodules even with unremarkable FNA.

\section{Data Availability}

All data generated or analyzed during this study are available from the corresponding author upon request.

\section{Ethical Approval}

This study protocol was reviewed and the need for approval was waived by the ethics committee at Palisades Medical Center Hackensack Meridian Health.

\section{Consent}

A written informed consent was obtained from the patient for publication of this case report and any accompanying images.

\section{Conflicts of Interest}

The authors declare that they have no conflicts of interest.

\section{Authors' Contributions}

Ayrton Bangolo searched the literature, wrote, and revised the manuscript. John Bukasa, Ali Atoot, Mohammad Jurri, Ashraf Mahmoud, Arthur Oliver, and Kunchang Song revised and edited the manuscript. Adam Atoot and Syed Sirajuddin revised and approved the final version and are the article's guarantors. All authors certify that they contributed sufficiently to the intellectual content and data analysis. Each author has reviewed the final version of the manuscript and approves it for publication.

\section{References}

[1] Y. C. Cozier, J. S. Berman, J. R. Palmer, D. A. Boggs, D. M. Serlin, and L. Rosenberg, "Sarcoidosis in black women in the United States," Chest, vol. 139, no. 1, pp. 144-150, 2011.

[2] R. P. Baughman, A. S. Teirstein, M. A. Judson et al., "Clinical characteristics of patients in a case control study of sarcoidosis," American Journal of Respiratory and Critical Care Medicine, vol. 164, no. 10, pp. 1885-1889, 2001.
[3] W. E. James, E. Koutroumpakis, B. Saha et al., "Clinical features of extrapulmonary sarcoidosis without lung involvement," Chest, vol. 154, no. 2, pp. 349-356, 2018.

[4] A. Vailati, C. Marena, L. Aristia et al., "Sarcoidosis of the thyroid: report of a case and a review of the literature," Sarcoidosis, vol. 10, no. 1, pp. 66-68, 1993.

[5] N. Porter, H. L. Beynon, and H. S. Randeva, "Endocrine and reproductive manifestations of sarcoidosis," QJM: International Journal of Medicine, vol. 96, no. 8, pp. 553-561, 2003.

[6] R. P. Baughman and E. E. Lower, "Sarcoidosis," in Harrison's Principles of Internal Medicine, 20e, J. L. Jameson, A. S. Fauci, D. L. Kasper, S. L. Hauser, D. L. Longo, and J. Loscalzo, Eds., McGraw-Hill Education, New York, NY, USA, 2018.

[7] A. M. Salih, S. M. Fatih, and F. H. Kakamad, "Sarcoidosis mimicking metastatic papillary thyroid cancer," International Journal of Surgery Case Reports, vol. 16, pp. 71-72, 2015.

[8] A. Antonelli, P. Fazzi, P. Fallahi, S. M. Ferrari, and E. Ferrannini, "Prevalence of hypothyroidism and Graves disease in sarcoidosis," Chest, vol. 130, no. 2, pp. 526-532, 2006.

[9] J. L. Winnacker, K. L. Becker, and S. Katz, "Endocrine aspects of sarcoidosis," New England Journal of Medicine, vol. 278, no. 9, pp. 483-492, 1968.

[10] L. Yassa, E. S. Cibas, C. B. Benson et al., "Long-term assessment of a multidisciplinary approach to thyroid nodule diagnostic evaluation," Cancer, vol. 111, no. 6, pp. 508-516, 2007.

[11] A. E. Samir, A. Vij, M. K. Seale et al., "Ultrasound-guided percutaneous thyroid nodule core biopsy: clinical utility in patients with prior nondiagnostic fine-needle aspirate," Thyroid, vol. 22, no. 5, pp. 461-467, 2012.

[12] C. H. Suh, J. H. Baek, C. Park, Y. J. Choi, and J. H. Lee, "The role of core needle biopsy for thyroid nodules with initially indeterminate results on previous fine-needle aspiration: a systematic review and meta-analysis," American Journal of Neuroradiology, vol. 38, no. 7, pp. 1421-1426, 2017. 